the oral cavity as a functional unit includes the salivary glands and temporo-mandibular joints they are concerned with these also. The fascination of dentistry lies not only in the challenge of its two major diseases and all the elaborate technical skills which may be deployed to repair the damage they cause, but also the wide range of other conditions which affect the oral cavity and its associated parts.

As Professor B. Cohen comments in his introductory remarks to the latest British Medical Bulletin, ${ }^{1}$ on the topic of research in dentistry, the incidence of dental caries has reached pandemic proportions in modern urban populations. Periodontal disease likewise is an almost universal scourge of dentate modern man. Few other conditions can rival them both for their high incidence and for the complacency with which the general public regard such widespread morbidity. Even more remarkable is the general reluctance of communities to make use of the public health and preventive measures which have been advocated for their control. Fluoridation of the water supply and the elimination of between-meal snacks, two simple measures, would greatly reduce the incidence of dental caries, as Hartles and Leach ${ }^{2}$ point out.

Both dental caries and periodontal disease are inherently difficult processes to investigate, and a few of the methods used are reviewed in the Bulletin. These papers give some insight into the many facets concerned: the structure of the tooth, the physiology of the gingiva, the composition of plaque, the epidemiology of caries, investigations into the way treatment may be organized and effected, and the effects on the tissues of some of the instruments used in treatment. Perhaps it is worth commenting that this last spring at the meetings in New York and London alone of the International Association for Dental Research over 1000 papers were read.

The 1921 Dentists' Act was drawn up to prevent unskilled dental practice, and the simplest way to do this was to forbid anyone except qualified dental and medical practitioners from undertaking procedures in the oral cavity. At the same time, however, the Act effectively prevented the development of properly trained ancillary help, such as has grown up over the years in general medicine. Fortunately, in the same year New Zealand started to train operating auxiliaries or "New Zealand dental nurses," showing the way that the graduate dentist could be freed from some of the less skilled duties in the practice of his profession. Allred and Hobdell's review ${ }^{3}$ of the development and enlargement of the dental team gives a glimpse of the dental practice of the future.

Though it is a specialized branch of medicine there has always been a separate undergraduate course for dentistry in Britain. In no small part this is due to the many technical skills which the general dental practitioner must possess; but the separate undergraduate course is also justified by the time taken to achieve an initial understanding of the diseases and the diverse pathological processes of the region. Based on this knowledge have grown the specialities of dentistry. Some of these, like oral and maxillofacial surgery, oral pathology, and oral medicine have moved closer to the main stream of medical practice. Not a few of their practitioners have both a dental and a medical qualification. Some have higher qualifications not only in dentistry but also in the appropriate related special field of medicine. With the growth of these specialties there has been an upsurge of interest in the treatment of facial deformity, neoplasia of the jaws and oral cavity, and diseases of the oral mucosa and salivary glands.

With the increasing complexity of modern therapeutics the importance for the general dental practitioner of a good understanding of general medicine is also increased. In order to facilitate this broader training in general medicine and to foster the continued growth of the specialties of dentistry, perhaps the time is ripe for a closer correspondence and a greater degree of interchangeability between the undergraduate courses in the two subjects.

The work surveyed in this British Medical Bulletin foreshadows the emergence of a dentist with rather different main interests. Were the general public to show a more responsible attitude towards their dental health and accept help from public health measures the incidence of caries and gross periodontal disease could be reduced. There would be, as a result of the same change in attitude, a more widespread desire for the restoration of those cavities which did occur, for professional control of periodontal disease, and for the correction of irregularity of the teeth and malocclusion. As the incidence of these conditions fell there would be every likelihood that the dental surgeon, supported by his operating assistants, could cope effectively with this demand for treatment. What is more, a liberally educated general dental practitioner would have time to join more closely with the specialist dental surgeon to deal with the many other problems of the maxillofacial region, some of which are no better understood today than caries and periodontal disease were a generation ago.

1 Cohen, B., British Medical Bulletin, 1975, 31, 99.

${ }^{2}$ Hartles, R. L., and Leach, S. A., British Medical Bulletin, 1975, 31, 137.

${ }^{3}$ Allred, H., and Hobdell, M. H., British Medical Bulletin, 1975, 31, 149

\section{Cardiac Implantation}

Total cardiac transplantation carries the obvious disadvantage that the recipient's future survival depends on the continued function of the transplant. Barnard and his team in Cape Town have now devised a new technique in which a donor heart is anastomosed in parallel to the patient's heart in such a way that the function of the patient's own heart is left intact. ${ }^{1}$ The two patients ${ }^{1}$ who have been treated by this technique were a man of 59 with ischaemic heart disease and advanced left ventricular failure and another man aged 47 who had rheumatic heart disease with aortic regurgitation and severe left ventricular myocardial dysfunction.

The donor heart is placed partly in the right pleural space and partly in the anterior mediastinum, next to the patient's own heart. Similar sized windows are made in the walls of both the donor and the recipient left atrium, and a side-to-side anastomosis is made between the two openings. An end-to-side anastomosis is made between two aortas; and another end-toside anastomosis is performed between the donor's pulmonary artery and the patient's right atrial appendage. The superior and inferior venae cavae of the donor heart having been tied off, its right ventricle has only its own coronary sinus return to handle, and this is returned into the patient's right atrium. The patient's right atrium and right ventricle receive both his own venous return and the coronary sinus return from the donor heart, and they function in a normal manner. Blood from the patient's pulmonary veins enters his own left atrium; it may then flow either into his own left ventricle or through the window into the donor left atrium to be pumped by the donor left ventricle. The total left ventricular work is therefore shared, and either the donor or the patient heart may handle most of the load.

The two hearts beat totally independently of each other, so the pulse is composed of a mixture of beats, some resulting from the activity of the donor and some from the patient's own 
left ventricle. The size of each pulse wave depends on the volume of blood which has entered the corresponding left ventricle, which in turn is determined by the timing of the ventricular diastoles in relation to each other and on the capacities of the two ventricles relative to each other. The atrial window functions as in congenital atrial septal defect with blood flowing preferentially to the ventricle which can at that instant accommodate the volume. No difficulty was found in creating room for the allografts, and there was no evidence of embarrassment of the function of the right lung.

Transplantation has usually been carried out for disorders, such as coronary artery disease, which primarily or exclusively affect the left ventricle and in which the right ventricle remains healthy. In such patients restoration of left ventricular function alone would result in alleviation of congestive failure. Patients in whom prolonged left ventricular failure has resulted in severe pulmonary hypertension have shown themselves to be unsuitable for cardiac transplantation because the normal donor right ventricle is unable to eject against the increased pulmonary vascular resistance. Retention of the hypertrophied right ventricle could provide an immediate advantage, even though in the long term the pulmonary hypertension might be expected to disappear following removal of its left-sided cause.

In patients who have diffuse myocardial disease such as congestive cardiomyopathy the recipient's right ventricle may be aided in similar fashion by joining the stump of the donor superior vena cava to the patient's own superior vena cava and then joining the donor's pulmonary artery to the patient's pulmonary artery. The technique carries the further advantage of allowing the surgeon to perform procedures on the patient's own heart which might improve its immediate or long term function. To this end the first patient was treated by excision of part of the fibrous infarcted wall of the left ventricle in an attempt to improve its performance. In the second patient the implantation was associated with exploration of an aortic prosthesis, repair of a small peripheral leak due to dehiscence of the suture line, and placement of a new silastic poppet.

One of the earliest signs of rejection is a drop in voltage in the implanted heart. ${ }^{2}$ In these two patients a fall in both the donor's and the patient's heart voltage was seen. A critical episode in the postoperative course of the first patient was an episode of ventricular fibrillation in his own heart on the 22nd day. On this occasion it was clear that the total cardiac output had dropped but the patient was not unduly disturbed. An electrocardiogram showed ventricular fibrillation in one heart and sinus rhythm in the other. Electrical defibrillation after lignocaine led to resumption of sinus rhythm in both hearts and thenceforward he was treated with prophylactic oral quinidine.

Placement of the patient's right heart in parallel with the donor heart may be advisable in patients like this one who are at risk from ventricular fibrillation, in order to maintain the venous return to the lungs; for otherwise ventricular fibrillation in the recipient heart would inevitably cause a fall in cardiac output however excellent the function of the donor left ventricle.

The assistance provided to the donor heart by the patient's own defective left ventricle ought to prove helpful during occasions when the donor heart might itself function suboptimally. This applies to the early postoperative period, with the inevitable anoxic damage caused by the delay time between death of the donor and re-establishment of a coronary circulation, and also during any episodes of rejection. ${ }^{2}$ The new operation does not preclude removal of the implant and replacement with another allograft should rejection or infection seriously damage the donor heart. Equally, if it seems desirable subsequently to remove the patient's own heart this too can be done relatively easily. Barnard and Losman suggest that the technique can be used either to provide permanent assistance, as in the two patients reported, or as a temporary device to be used in the same sort of situations as balloon pump counterpulsation after cardiac surgery or in cardiogenic shock.

1 Barnard, C. N., and Losman, J. G., South African Medical Fournal, 1975, 49, 303.

2 Graham, A. F., et al., Transplantation Proceedings, 1974, 6, Supplement 1, 17.

\section{Ampicillin Rashes}

Doctors are often slow to recognize drug eruptions, and rashes tend to be attributed to a disease rather than its treatment. Those due to ampicillin are the exception. They are so familiar now that this antibiotic may even be blamed more often than is just. If infectious mononucleosis is excluded, ampicillin rashes occur in from $3 \%$ to $8 \%$ of patients taking it. A multicentre survey reported ${ }^{1}$ that in a prospective study rashes were seen in $7.3 \%$ of 933 patients. However, the overall incidence in a retrospective study ${ }^{2}$ of 13638 patients reported throughout the world was only $2 \cdot 8 \%$.

It seems that the likelihood of developing an ampicillin rash may be influenced by the clinical state of the patient. Nearly all those with infectious mononucleosis, if given a total dose of $1.5 \mathrm{~g}$ or more of ampicillin, will get a rash. $^{3}{ }^{7}$ An increased incidence has also been reported in patients with chronic lymphatic leukaemia, ${ }^{4}$ cytomegalovirus infection, ${ }^{5}$ viral respiratory tract infections, ${ }^{1}$ and hyperuricaemia treated with allopurinol. ${ }^{6}$ Females are more often affected than males. ${ }^{1}$ The incidence of rashes may rise with the dose of

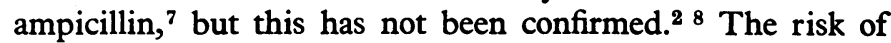
developing a rash with ampicillin is greater than with other penicillins. ${ }^{9}$

Though urticarial ${ }^{1}$ and rarely anaphylactic ${ }^{11}$ reactions have been described, the most common ampicillin rash is erythematous and macular or maculopapular. ${ }^{1}$ It usually appears about a week after the start of treatment and lasts for a similar period. Confusion may occur when the rash appears several days after treatment has been stopped. ${ }^{7}$ The trunk is often affected first, but soon there is spread to the limbs, particularly the fronts of the knees and backs of the elbows. ${ }^{1}$ Itching is common but not unduly severe. Purpura, joint swelling, and lymphadenopathy are rare variants. ${ }^{1}$ There are reports of the rash fading while treatment continues or failing to reappear if the patient is given the drug again. ${ }^{10}$

Knudsen ${ }^{2}$ found no evidence that the rarer urticarial type of rash was more common with ampicillin than with other penicillins but suggested that the maculopapular rash was specific for ampicillin and did not indicate true penicillin hypersensitivity. ${ }^{2}$ This view is supported by the finding that serum penicilloyl-specific antibodies are neither quantitatively nor qualitatively different in patients with or without the maculopapular rash. ${ }^{12}$ Webster and Thompson ${ }^{12}$ also failed to show positive skin reactions to the major and minor antigenic determinarts of penicillin when they tested patients with ampicillin rashes of the maculopapular type.

Commercially available ampicillin contains traces of protein impurities, which are difficult to eliminate in its manufacture. ${ }^{13}$ They are antigenic, and injection of the isolated protein 